\title{
OESOPHAGUS
}

\section{Eosinophilic oesophagitis: a novel treatment using Montelukast}

\author{
S E A Attwood, C J Lewis, C S Bronder, C D Morris, G R Armstrong, J Whittam
}

See end of article for authors' affiliations ......................

Correspondence to: Mr S E A Attwood, Department of Upper Gastrointestinal Surgery, Hope Hospital, Stott Lane, Manchester M6 8HD, UK chris.lewis@hope.srht.nhs.uk

Accepted for publication 26 July 2002

\begin{abstract}
Background: Eosinophilic oesophagitis is a rarely diagnosed condition involving eosinophil infiltration of the oesophageal mucosa and creating significant symptoms of dysphagia. Failure to diagnose this disorder relates to reluctance to biopsy an apparently normal oesophagus. This is essential for histological diagnosis. To date, treatment success has been achieved only with corticosteroids. We describe here the use of an eosinophil stabilising agent Montelukast for the symptomatic relief of these patients.

Patients and methods: Twelve patients have been identified with this condition in our unit since 1995, after thorough investigation of their dysphagia. We commenced eight of these patients on the leukotriene receptor antagonist Montelukast to symptomatically improve their swallowing while avoiding the use of long term corticosteroids.

Results: Many of these patients had been previously misdiagnosed, and therefore inappropriately and unsuccessfully treated for an extensive period prior to referral to our unit. All patients were unresponsive to acid suppression therapy alone but showed improvement in their swallowing on Montelukast. Six of eight reported complete subjective improvement, five patients remaining completely asymptomatic on a maintenance regimen.

Conclusions: Eosinophilic oesophagitis is a disease that is often misdiagnosed due to lack of awareness and reluctance of clinicians to biopsy an apparently normal oesophagus in dysphagic patients, and therefore obtain a histological diagnosis. Investigation of these patients adds further evidence to this condition being a separate pathological state from gastro-oesophageal reflux and eosinophilic enteritis. Montelukast has been found to be of significant help in the symptomatic control of these patients while avoiding long term corticosteroids use.
\end{abstract}

A twood et al called attention to eosinophilic oesophagitis (EO) as a distinct clinical condition in 1993. In this disease an intense eosinophilic infiltration is seen in the oesophagus of dysphagic patients. ${ }^{1}$ Previously, eosinophils in the oesophagus were regarded as an incidental component of gastro-oesophageal reflux disease $\mathrm{e}^{2}$ or a variant of eosinophilic gastroenteritis. ${ }^{3}{ }^{4}$ However, the distinct clinical syndrome of EO is not usually seen with either of these. Most recent literature refers to EO in children but the adult phenomenon may escape diagnosis due to lack of awareness of this as a separate pathology. ${ }^{15}$

The hallmark of this condition is intermittent and often painful dysphagia which may become constant as the disease progresses. Thorough investigations often show essentially normal or mildly abnormal oesophageal endoscopy, $\mathrm{pH}$, and manometry studies which are not consistent with the severity of symptoms described. Mechanical obstruction is usually absent and, unless a biopsy is taken, these patients are found to be a diagnostic and management enigma.'

Management of these patients has been made difficult due to diagnostic problems and a poor understanding of the disease pathogenesis. Acid suppression has no effect despite the theory that this condition is secondary to gastrooesophageal reflux. ${ }^{2}$ Antihistamine medications have been used but with no reported success. Oesophageal dilatation has been undertaken for purely symptomatic relief but unfortunately this can be very uncomfortable and provides only short term relief. ${ }^{1}$ Corticosteroid therapy has been described with some success. ${ }^{56}$ However, symptoms soon relapse after ceasing therapy, and the significant side effect profile of long term steroid therapy greatly limits their value in this setting. ${ }^{5}$

In the paediatric setting, this condition is far more widely recognised. The standard therapy regimens consist of an elimination diet and corticosteroids. ${ }^{7}$ However, this is an arduous undertaking and would probably be unacceptable to the adult population.

A recent case report by Schwartz and colleagues ${ }^{8}$ describes the use of Montelukast (Singulair; Merck Sharp and Dohme Ltd, UK) in the treatment of steroid dependent eosinophilic gastroenteritis. Montelukast is a leukotriene receptor antagonist (LTRA) which actively and selectively blocks the leukotriene D4 receptor.' It was originally licensed as a cysteinyl leukotriene antagonist for use in asthma. We have been using Montelukast in the treatment of EO in a similar way for some years. This report describes our clinical experience of patients with EO treated in this way.

\section{MATERIALS AND METHODS}

Since 1995 we have identified 12 patients referred to our unit with EO. Until the advent of Montelukast, and its wide availability in 1998, a variety of treatment regimens had been tried with differing success. Treatment of these patients was primarily adjusted according to the severity of symptoms. Those with mild symptoms were commenced on either cinnarizine (Stugeron; Janssen-Cilag Ltd, UK) 15-30 mg three times daily or sodium cromoglycate (Nalcrom; Pantheon Healthcare Ltd, UK) $200 \mathrm{mg}$ four times daily. Patients with more severe symptoms were commenced on oral corticosteroid therapy and showed significant symptom relief. One of three patients showed significant improvement on cinnarizine. One patient's

Abbreviations: EO, eosinophilic oesophagitis; LTRA, leukotriene receptor antagonist; GORD, gastro-oesophageal reflux disease. 


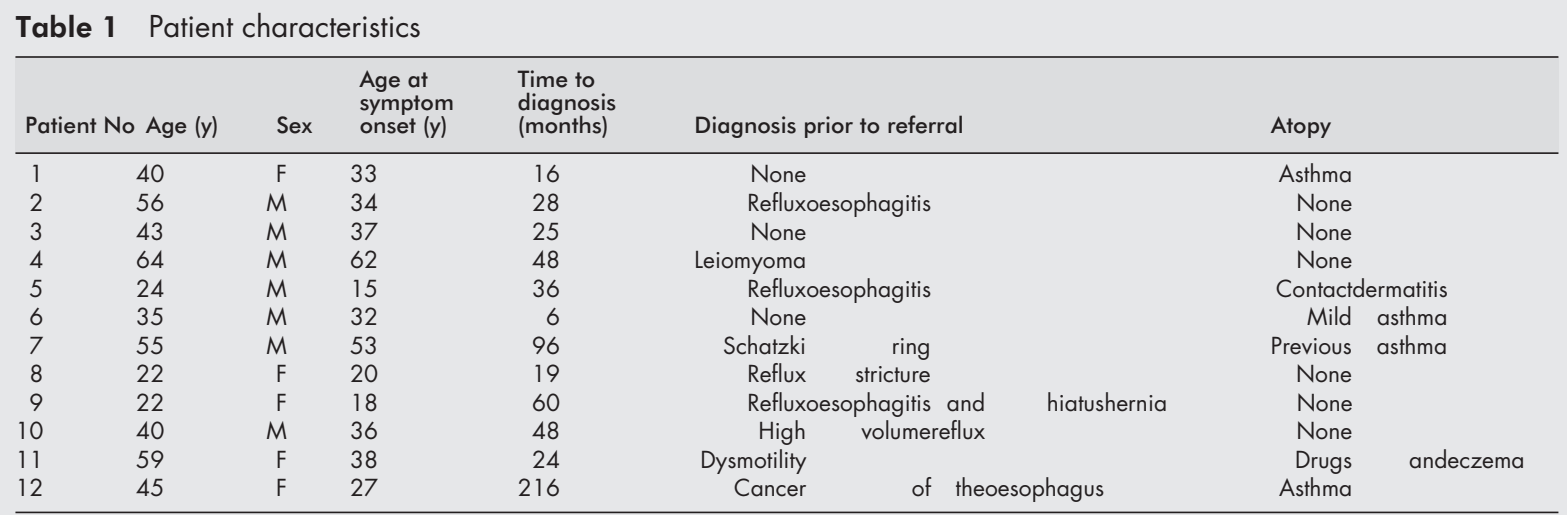

Table 2 Patient symptoms and previous investigations

\begin{tabular}{|c|c|c|c|c|c|c|c|}
\hline $\begin{array}{l}\text { Patient } \\
\text { No }\end{array}$ & Dysphagia & $\begin{array}{l}\text { Precipitating } \\
\text { agent }\end{array}$ & Odynophagia & a Regurgitation & Heartburn & Bolus obstruction & Previous investigations \\
\hline 1 & Intermittentbimonthly & Solids & +++ & - & + & - & - \\
\hline 2 & Intermittent weekly & Solids andliquids & $s+$ & + & - & - & OGD+bariumswallow \\
\hline 3 & Intermittent biweekly & Solids & - & - & + & - & OGD \\
\hline 4 & Constant & Solids & - & + & - & - & OGD \\
\hline 5 & Intermittent weekly & Meat & + & + & - & $\times 3$ & OGD \\
\hline 6 & Intermittent weekly & Drybread & + & - & - & - & - \\
\hline 7 & Intermittent/total annually & Meat & - & - & - & $\times 1$ & OGD+dilatation \\
\hline 8 & Constant & Anything & - & - & - & $\times 1$ & OGD+bariumswallow \\
\hline 9 & Constant & Anything & ++ & - & + & - & OGD \\
\hline 10 & Intermittent weekly & Solids & ++ & + & + & - & - \\
\hline 11 & Intermittent monthly & Solids & - & + & + & - & OGD \\
\hline 12 & Intermittent daily & Solids & + & - & + & - & OGD+bariumswallow \\
\hline
\end{tabular}

+ , mild; ++ , moderate; +++ , severe; - , absent.

OGD, oesophagogastroduodenoscopy.

symptoms spontaneously resolved while another was helped by myotomy. ${ }^{10}$

Patients who previously showed improvement in symptoms with steroids and those with poor relief from other regimens were commenced on Montelukast in an attempt at symptom control without the need for long term corticosteroid therapy.

Investigation of patients with EO was standardised. Each patient underwent oesophagogastroduodenoscopy (Fujinon Series EG410-HR and EG410D gastroscopes; Fujinon Inc. USA) and multiple biopsies using the $2.2 \mathrm{~mm}$ Boston Scientific Microvasive Radial Jaw 3 with Needle (Boston Scientific Intl, France), both at diagnosis and again 4-9 months following commencement of Montelukast. All were performed following fully informed consent under sedation using 4-8 mg midazolam (Hypnovel; Roche Products Ltd, UK). Formalin fixed biopsies were then processed in paraffin sections, stained with haematoxylin and eosin, and examined under high power view $\left(\times 40\right.$ : field area $\left.0.166 \mathrm{~mm}^{2}\right)$ in random orientation by a single pathologist.

Manometry and $\mathrm{pH}$ studies were undertaken on all patients to identify any motility disorders or reflux. Stationary manometry was performed using a five channel solid state catheter (Gaeltec Ltd, Scotland, UK), while 24 hour pH was measured $5 \mathrm{~cm}$ above the lower oesophageal sphincter using an antimony electrode on a single channel probe (Medtronic Functional Diagnostics, Denmark) and recorded on a Digitrapper III (Synectics Medical, Sweden). Acid exposure and DeMeester score were then calculated on Gastrosoft computer software (Gastrosoft Inc., USA). Bile reflux was tested in one patient using Bilitec 2000 (Synectics Medical).

The dose was adjusted according to tolerance and symptoms. Initial treatment was with Montelukast $10 \mathrm{mg}$ daily but was increased if required up to a total of $100 \mathrm{mg}$ daily. This starting dose was the same as that used for patients with severe asthma in hospital. Once symptom relief had been achieved, the dose was then reduced to maintenance levels where symptom control was retained. In all patients this was between a total dose of $20 \mathrm{mg}$ and $40 \mathrm{mg} /$ day.

\section{Patients}

Median age of patients with EO was 40 years (range 22-64; interquartile range 35-55). Onset of symptoms was at a median age of 33 years (range 15-62). Median length of time patients had symptoms prior to referral to our unit was 36 months (range six months to 18 years) (table 1). Eight patients were referred directly from gastroenterologists, one from a neurologist, one from a cardiothoracic surgeon, and two from general practice. Two patients had also seen ear, nose, and throat surgeons prior to attending our department.

All patients presented with dysphagia as their primary symptom. They did however have a variety of other symptoms, notably odynophagia in seven patients, and a further potential allergic disorder in six (table 2).

At referral, nine patients had previously had a flexible gastroscopy, two of these had also had rigid oesophagoscopy, and three had undergone a barium swallow. The diagnoses given at referral were oesophageal dysmotility in one patient, acid or alkali reflux with or without stricture in six patients, and suspected oesophageal carcinoma in another (table 1).

\section{RESULTS}

A specific pattern of mucosal change was observed at endoscopy in nine patients. Two patients had concentric rings (fig 1) and four had linear furrows (fig 2) in the mucosa. In 


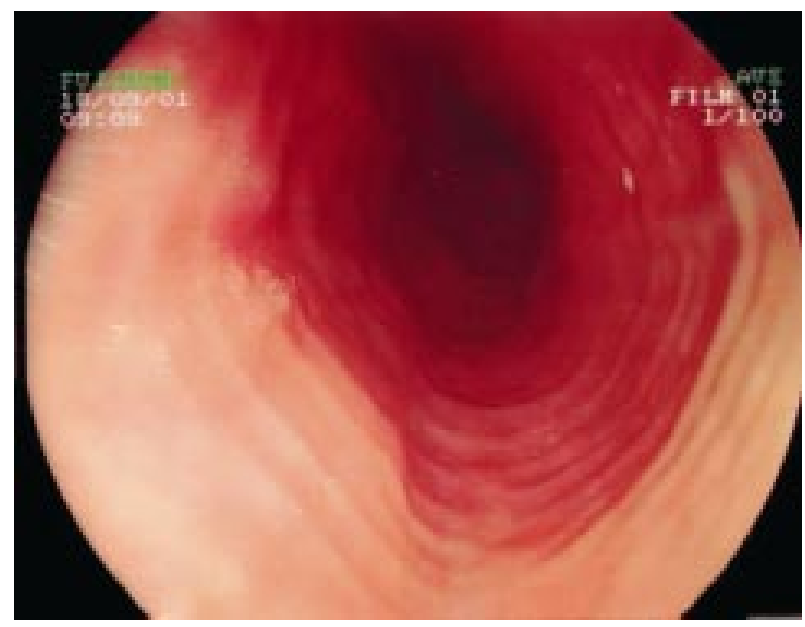

Figure 1 Endoscopic view of a dysphagic patient with eosinophilic oesophagitis, illustrating clearly a concentric ring pattern.

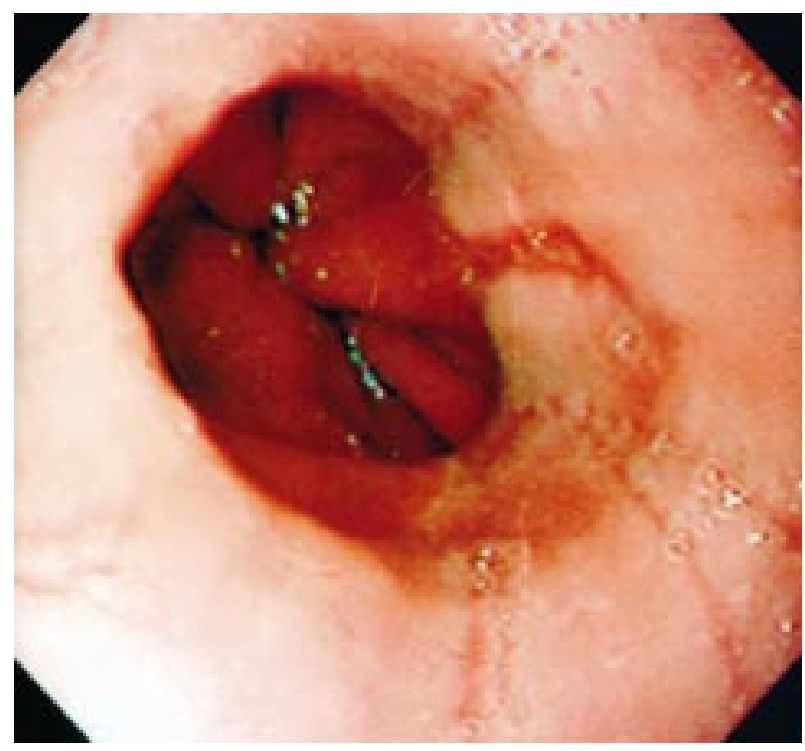

Figure 2 Endoscopic view of a dysphagic patient with eosinophilic oesophagitis, illustrating linear furrows.

three patients there were both furrows and rings, creating a cobblestone effect ( fig 3). Two patients had a macroscopically normal oesophagus while one was seen to have apparent extrinsic compression caused by an intramural leiomyoma.

Biopsies were examined and formal eosinophil counts were taken using an eyepiece graticule. From this an eosinophil density per $\mathrm{mm}^{2}$ was calculated. Median density of eosinophils was $342.5 / \mathrm{mm}^{2}$ (range 115-871.3), equivalent to 56.9 per high power field. All eosinophils were concentrated in the intraepithelial layer except for one patient who had eosinophils present in the muscle layer but had few in the intraepithelial layer $\left(20.3\right.$ per $\left.\mathrm{mm}^{2}\right)$. This patient had a myotomy and a biopsy which was taken at thoracotomy. All of the remaining patients had endoscopic biopsies and therefore no information on eosinophil infiltration of the muscle layer was available. On inspection of the biopsy slides it was evident that eosinophil infiltration was non-uniform. There was marked variation within each section, and microabscesses were also present in the intraepithelial layer. Treatment with Montelukast for more than four months did not change the density of eosinophils.

$\mathrm{pH}$ and manometry studies showed that all but one patient had no evidence of reflux, with median DeMeester scores of 7.4 (range $0.9-10$ ), median oesophageal exposure time to a

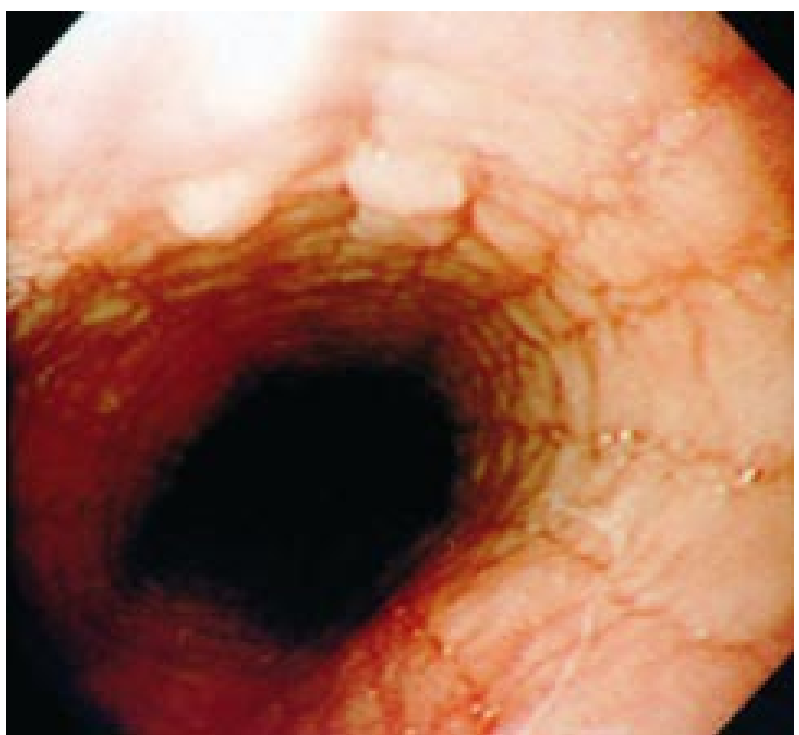

Figure 3 Combination of furrows and rings creating a cobblestone effect seen in eosinophilic oesophagitis at endoscopy.

$\mathrm{pH}<4$ of $1.5 \%$ (range $0.3-2.6 \%$ ), and a median lower oesophageal sphincter barrier pressure of $20 \mathrm{~mm} \mathrm{Hg}$ (range 5-39). One patient presented with significant reflux and a Schatzki ring, which required dilatation. His DeMeester score was 20; the time for oesophageal acid exposure was 5.9\%, and his lower oesophageal sphincter pressure was $9 \mathrm{~mm} \mathrm{Hg}$. This patient appeared to have dual pathologies, which was evident when he required acid suppression therapy, in addition to Montelukast, for symptom control.

Eight patients have been commenced on Montelukast. Two had previously responded to corticosteroids but were reluctant to remain on this therapy for the long term. Every patient had been treated with a proton pump inhibitor previously, and two with a combination of antihistamines and motility agents (table 3), all without success. Cessation of the acid suppression therapy showed no detrimental effect in 11 of 12 patients. Montelukast has restored a comfortable swallow in all those treated, except for two patients who still have some residual discomfort despite experiencing improved symptomatology.

Montelukast was tolerated well at $30 \mathrm{mg}$ daily but five patients required an increased dose. Seven of the eight showed complete subjective improvement in dysphagia symptoms with a maintenance dose of $20-40 \mathrm{mg}$ daily. The eighth had moderate improvement at $30 \mathrm{mg}$ but could not tolerate the $40 \mathrm{mg}$ dose due to myalgia and nausea. He did however choose to remain on the medication (table 3 ).

These patients have now been treated with Montelukast for a median of 14 months (range 4-28). No relapse of symptoms has been described while continuing on the medication but six patients have had recurrence within three weeks of cessation or reduction in medication. Three patients still experience other symptoms occasionally, such as odynophagia. Several side effects were documented, most notably nausea in four patients and myalgia in one, but there was a significant subjective improvement throughout the group. Of this series of patients, eight remain on Montelukast, one is still on cinnarizine, and three require no further medication. One has undergone surgery and has been reported on previously. ${ }^{10}$

\section{DISCUSSION}

Eosinophilic oesophagitis is a distressing condition of dysphagia which responds well to Montelukast. The difficulties related to diagnosis are primarily due to failure to appreciate subtle mucosal changes at endoscopy and therefore failure to biopsy where no significant pathology is visualised. EO 
Table 3 Treatment results

\begin{tabular}{|c|c|c|}
\hline Patient No & Treatment & Improvement \\
\hline \multirow[t]{3}{*}{1} & Cinnarizine & - \\
\hline & Cromoglycate & - \\
\hline & Dilatation & - \\
\hline 2 & Cinnarizine & +++ \\
\hline 3 & None & Spontaneous resolution \\
\hline 4 & Myotomy & ++ \\
\hline \multirow[t]{5}{*}{5} & Cinnarizine & - \\
\hline & Chlorpheniramine & - \\
\hline & Cromoglycate & - \\
\hline & Corticosteroids & +++ \\
\hline & Montelukast $10 \mathrm{mg}$ tds & ++ \\
\hline \multirow[t]{2}{*}{6} & Corticosteroids & +++ \\
\hline & Montelukast10mgqds & +++ \\
\hline \multirow[t]{3}{*}{7} & Omeprazole & - \\
\hline & Dilatation & - \\
\hline & Rabeprazole+Montelukast10mgqds & +++ \\
\hline \multirow{3}{*}{9} & Montelukast $10 \mathrm{mg}$ tds & +++ \\
\hline & Cisapride+lanzoprazole & - \\
\hline & Montelukast $10 \mathrm{mg}$ tds & +++ dysphagia,odynophagia remains \\
\hline \multirow[t]{2}{*}{10} & Lanzoprazole & - \\
\hline & Montelukast $10 \mathrm{mg}$ tds & +++ \\
\hline 11 & Montelukast10mg tds & othersymptomsremain \\
\hline \multirow[t]{2}{*}{12} & Omeprazole & - \\
\hline & Montelukast10mg bd & +++ \\
\hline
\end{tabular}

is only identified histologically using oesophageal biopsies where high densities of eosinophils ( $>20$ /high powered field) are found infiltrating the mucosa. ${ }^{1}$ From our group of patients it is also clear that there is still a significant problem with the diagnosis of EO. Median time from onset to diagnosis was 33 months, which should be improved with greater understanding and awareness of this separate disease entity.

The aetiology of EO is not fully understood. It has been theorised that infiltration of eosinophils into the oesophageal mucosa is the oesophageal manifestation of allergic eosinophilic gastroenteritis, ${ }^{34}$ or is this condition associated with reflux disease? ${ }^{2}$ Although response to steroids has been reported to be significant in some patients, as it was in our patient group, they do not manifest any other signs or symptoms of eosinophilic gastroenteritis, suggesting that the two pathologies may not be linked, as previously suggested. ${ }^{34}$ It is also very clear that these patients do not respond well to acid suppression therapy and that histological and endoscopic findings, although similar, are not completely consistent with the reflux aetiology postulated by Lee. ${ }^{2}$

Reflux as a causative mechanism is still a widely held belief. Morrow et al's ${ }^{11}$ paper postulated the disease phenomenon of so-called "ringed oesophagus" as primarily a result of gastrooesophageal reflux disease (GORD). This ringed oesophagus, by definition, has all the hallmarks of EO. The connection between the two supposedly different pathologies has not been made as $\mathrm{pH} / \mathrm{manometry}$ studies showed GORD in only two patients in their study. ${ }^{11}$ In their series of 19 patients, all presented in the same way as our group, with progressive dysphagia. Half of the patients had onset in the same age range and all had concentric rings, very similar to those found in five patients from our group with proven EO. Only 11 patients had available biopsies from endoscopy, highlighting the lack of routine biopsy of all patients with dysphagia of unknown cause. All showed significant infiltration of eosinophils but quantitative measurements were omitted from the results. In our study only one patient was shown to have significant acid exposure on 24 hour monitoring. In their paper, Morrow et al treated a single patient with corticosteroids. The patient made a significant improvement, as would be expected in a patient with EO. The remainder however were treated with repeated and often painful dilatation along with acid suppression.
Somewhat surprisingly they report subjective improvement in symptoms despite unproved reflux and even absence of reflux symptomatology in nearly $50 \%$ of patients. Morrow et al conclude by advocating repeat dilatation and acid suppression therapy in a patient group with identical presentation and similar histological findings to our own, a view we do not from our experience share.

Of our patients, only $50 \%$ had a history of atopy (table 1$).^{5}$ Similarly, there was no improvement with the use of antihistamines in the treatment of symptoms, except in one patient, which suggests a different pathogenesis from an allergenic cause. Our patients showed no symptoms as young children. The onset of the disease appeared on the whole later in life, implicating a separate pathological state than the more widely reported paediatric EO. This again is attributed to reflux disease in the main which does not fit our patient group. $^{67}$

The difficulty of diagnosis through lack of biopsy has caused one of our patients to be referred to a psychiatrist for what the clinician believed to be a psychogenic disorder. A second patient's dysphagia was thought to be a manifestation of his severe depression, and was again referred to a psychiatrist. These patients have been discharged from the care of the mental health team following confirmed true diagnosis, and have returned to normal social function.

It is clear that this pilot use of Montelukast for the symptomatic treatment of EO shows that there is an effective treatment and an alternative to long term corticosteroid therapy. Montelukast selectively blocks the D4 receptor of cysteinyl leukotrienes present in eosinophils. All cysteinyl leukotrienes are arachidonic acid derived inflammatory mediators, ${ }^{12}$ which are important in asthma. ${ }^{13}$ The actions of leukotrienes include: eosinophil attraction and migration, powerful constriction of smooth muscle, airway oedema, mucous hypersecretion, and reduction in ciliary motility. ${ }^{34}$ They are predominantly released by eosinophils, basophils, and mast cells. ${ }^{14}{ }^{15}$ By blocking the D4 receptor the inflammatory action of the eosinophil cell is reduced. Eosinophils are not removed, there actions are simply negated, which was clearly seen in our repeated biopsy results.

Three published randomised control trials of Montelukast, for both chronic and exercise induced asthma, have shown 
significance in either symptom improvement or reduction in the requirement of other therapies, such as corticosteroids. ${ }^{16-18}$ The current guidelines allow Montelukast and other LTRAs to be used in asthma that is difficult to control, despite the use of corticosteroids, and not as firstline therapy. ${ }^{19}$

In conclusion, we have introduced a new potential treatment for EO. The study highlights the problems of diagnosis and lack of awareness and understanding of the aetiology and pathogenesis of the disease, and produces new evidence to refute the claims that this is a pathological state caused by gastro-oesophageal reflux or atopy. The use of Montelukast in this instance showed improvement of symptoms, although subjective, throughout the group. However, we have treated only a small number of patients and further randomised control trials, using this as a pilot study, are required to assess the full benefits of Montelukast in this condition. We stress the importance of oesophageal biopsies in all patients with dysphagia. This should be part of the standard protocol for investigation of these patients and the only way that the diagnosis of EO can be made.

\section{Authors' affiliations}

S E A Attwood, C J Lewis, C S Bronder, C D Morris, Department of Upper Gastro-intestinal Surgery, Hope Hospital, Salford Royal Hospitals NHS Trust, Manchester, UK

G R Armstrong, Department of Pathology, Hope Hospital, Salford Royal Hospitals NHS Trust, Manchester, UK

J Whittam, Pharmacy Department, Hope Hospital, Salford Royal Hospitals NHS Trust, Manchester, UK

\section{REFERENCES}

1 Attwood SEA, Smyrk TC, Demeester TR, et al. Esophageal eosinophilia with dysphagia. A distinct clinico-pathological syndrome. Dig Dis Sci 1993;38:109-16.
2 Lee RG. Marked eosinophilia in esophageal mucosal biopsies. Am J Surg Pathol 1986;10:75-86.

3 Dobbins JW, Sheahan DG, Behar J. Eosinophilic gastroenteritis with oesophageal involvement. Gastroenterology 1977;72:1312-16.

4 Goldman $\mathbf{H}$, Proujansky R. Allergic proctitis and gastroenteritis in children: clinical and mucosal biopsy features in 53 cases. Am J Surg Pathol 1985:9:475-9.

5 Straumann HB, Spichtin R, Bernolli J, et al. Idiopathic eosinophilic oesophagitis. Swiss Med Wkly 1994;124:1419-29.

6 Liacouras CA, Wenner WJ, Brown K, et al. Primary eosinophilic esophagitis in children: successful treatment with oral corticosteroids. $J$ Pediatr Gastroenterol Nutr 1998;26:468-71.

7 Orenstein SR, Shalaby TM, DiLorenzo C, et al. The spectrum of eosinophilic esophagitis beyond infancy: A clinical series of 30 children. Am J Gastroenterol 2000;95: 1422-30.

8 Schwartz DA, Pardi DS, Murray JA. Use of Montelukast as steroid-sparing agent for recurrent eosinophilic gastroenteritis. Dig Dis Sci 2001;46:1787-90.

9 Markham A, Faulds D. Montelukast. Drugs 1998;55:251-6.

10 Morris CD, Attwood SEA, Armstrong GR, et al. Diffuse esophageal leiomyomatosis with local dense eosinophilic infiltration. Dis Esoph 2002; 15:85-7.

11 Morrow BJ, Vargo JJ, Goldblum JR, et al. The ringed esophagus: histological features of GERD Am J Gastroenterol 2001;96:984-9.

12 Samuelsson B. Leukotrienes: mediators of immediate hypersensitivity reaction and inflammation. Science 1983; 220:568-75.

13 Sampson A, Holgate S. Leukotriene modifiers in the treatment of Asthma. BMU 1998:316:1257-8.

14 British National Formulary 3.3. Montelukast and zafirlukast in asthma. Drugs Ther Bull 1998;36:65-9

15 Hay DWP. Pharmacology of leukotriene receptor antagonists: more than inhibitors of bronchoconstriction. Chest 1997;111(suppl 2):35-45.

16 Noonan MJ, Chervinsky P, Brandon M, et al. Montelukast, a potent leukotriene receptor antagonist, causes dose-related improvements in chronic Asthma. Eur Respir J 1998;11:1232-9.

17 Reiss TF, Chervinsky P, Dockhorn R, et al. Montelukast, a once daily leukotriene receptor antagonist, in the treatment of chronic asthma. A multi-centre, randomised, double blind trial. Arch Intern Med 1998;158:1213-20.

18 Leff JA, Busse WW, Pearlman D, et al. Montelukast, a leukotriene receptor antagonist, for treatment of mild asthma and exercise induced bronchoconstriction. N Engl J Med 1998;339:147-52.

19 British Thoracic Society. The British guidelines on Asthma management 1995 review and position statement. Thorax 1997;52(suppl 1):S1-21. 